\title{
Intestinal microbiota and functional characteristics of black soldier fly larvae (Hermetia illucens)
}

\author{
Yuan Zhineng ${ }^{1,2}$, Ma Ying ${ }^{1,3}$, Tang Bingjie ${ }^{1}$, Zeng Rouxian and Zhou Qiang ${ }^{1 *}$
}

\begin{abstract}
Purpose: Black soldier fly transforms organic waste into insect protein and fat, which makes it valuable for ecological utilization. This process is associated with the intestinal microbiota. This research was developed to determine the type and functional characteristics of intestinal microbiota present in black soldier fly larvae.

Methods: In this research, metagenomics has been used to study black soldier fly larvae gut bacteria, which involves the high abundance of the gut microbe advantage bacterium group, the impact, and the physiological functions of the microbiota. Furthermore, intestinal bacteria and their related functions were investigated by bioinformatics analysis to evaluate potential microbial strains that may be used to improve feed utilization efficiency in factory farming.

Result: The results showed that black soldier fly larvae's intestine contains more than 11,000 bacteria. The high relative abundance of group W (larvae fed with 75\% wheat bran and 25\% soybean powder) may promote feed utilization efficiency, whereas high relative abundance of group T microbiota (larvae fed with 75\% wheat bran and $25 \%$ soybean powder supplemented with $1 \%$ tetracycline) may play an important role in black soldier fly larvae survival.

Conclusion: The gut bacteria in black soldier fly larvae were involved in polysaccharide biosynthesis and metabolism, translation, membrane transport, energy metabolism, cytoskeleton, extracellular structures, inorganic ion transport and metabolism, nucleotide metabolism, and coenzyme transport physiological processes. The 35 significant differential microbes in group W may have a positive impact on feed utilization and physiological process.
\end{abstract}

Keywords: Hermetia illucens, Intestinal bacteria, Utilization efficiency, Metagenomics

\section{Background}

Black soldier fly Hermetia illucens (Diptera: Stratiomyidae) larvae are commonly used to recycle organic waste (van Huis 2013). They feed on organic waste including livestock manure (Rehman et al. 2017; Beskin et al. 2018), food waste (Nguyen et al. 2015), and organic waste (Li et al. 2011). The black soldier fly is currently used as a tool for waste transformation and utilization, but few

\footnotetext{
* Correspondence: Isszhou@mail.sysu.edu.cn

'The School of Life Sciences, Sun Yat-sen University, Guangzhou 510275, China

Full list of author information is available at the end of the article
}

researches consider diverse protocols to improve its waste utilization efficiency. Intestinal microorganisms are involved in several insect functions, including nutritional coordination (Douglas 1998), defense against plant toxins (Hammer and Bowers 2015), physiological response (Basset et al. 2000; Li et al. 2016), life span increase (Hoyt et al. 1971), influence on the development and reproductive potential (Gavriel et al. 2011; Prado and Almeida 2009), and detoxifying of specific foods (Hehemann et al. 2010), among others. Collectively, insect intestinal microorganisms play a crucial role in the growth and development of insects. Other studies

(c) The Author(s). 2021 Open Access This article is licensed under a Creative Commons Attribution 4.0 International License, which permits use, sharing, adaptation, distribution and reproduction in any medium or format, as long as you give appropriate credit to the original author(s) and the source, provide a link to the Creative Commons licence, and indicate if changes were made. The images or other third party material in this article are included in the article's Creative Commons licence, unless indicated otherwise in a credit line to the material. If material is not included in the article's Creative Commons licence and your intended use is not permitted by statutory regulation or exceeds the permitted use, you will need to obtain permission directly from the copyright holder. To view a copy of this licence, visit http://creativecommons.org/licenses/by/4.0/ 
have shown that bacteria in insects' gut play an important role in promoting food utilization. In this regard, gut bacteria of wood-feeding higher termite promote cellulose and xylan hydrolysis (Warnecke et al. 2007), whereas Odontotaenius disjunctus intestinal microbiota contributes to lignocellulose decomposition (Ceja-Navarro et al. 2019). Studies of black soldier fly larvae gut microbes include antibacterial peptide active substances extraction (Park et al. 2015), intestinal specific microbiota (Xie 2010), conserved microbiota analysis (Shelomi et al. 2020), and active enzymes analysis and identification (Kim et al. 2011; Lee et al. 2014, 2016). A recent review evaluated black soldier fly larva microbial community and prospected its feasibility to utilization improvement (De Smet et al. 2018). Furthermore, a relevant research finished an in-depth study to classify the positive influence of the gut microbiota of black soldier fly larvae (Bruno et al. 2019). However, there is scarce research focused on biological function analysis of black soldier fly larvae's gut microbiota. In addition, no research has been performed to search for microbiota that enhance feed utilization efficiency. It then becomes necessary to develop research to better understand the black soldier fly larvae's microbiota characteristics and functions.

Metagenomics research is a useful tool in gut microbe research, whose information is based on sequencing data (Furrie 2006). Based on the development of this technology, many unculturable microbes have been studied and analyzed (Handelsman 2004). In this concern, metagenomics research has expanded the mining of microbial community structure and function in the environment. To evaluate the black soldier fly larvae's intestinal bacteria biological function and screen the potential microbial strain that may be exploited to improve feed utilization efficiency in factory farming, such bacteria were systematically analyzed through metagenomics.

\section{Results}

\section{Data quality assessment}

Raw reads obtained from contig sequencing were assembled with the software MEGAHIT (Gurevich et al. 2013) in default parameter. Contig sequences shorter than 300 bp were discarded, and the assembly results were evaluated by QUAST (Zhu et al. 2010) with the default parameter. The results demonstrated that the number of contig in different experimental groups was about 600 , 000 and greatly varied in length. The N50 length was about $1000-1200 \mathrm{bp}$ with the alignment rate exceeding 95\% (Table 1).

MetaGeneMark (Fu et al. 2012) was used to identify coding regions in the genome with default parameters. Gene prediction was conducted according to the assembled contigs. The number of genes in different samples was about 500,000. The average gene length measured in each sample was in the range of 260 to $340 \mathrm{bp}$. The Cd-hit software (version 4.6.6) was used to remove the redundancy with default parameters; the similarity threshold was set at $95 \%$ and the coverage threshold at $90 \%$ (Fu et al. 2012). It was concluded that there were 2 , 168,041 non-redundant genes obtained in this sequence process, with an average length of $280 \mathrm{bp}$ (Table 2).

\section{Species information statistics}

The species composition and relative abundance of the samples were obtained by comparing the above nonredundant genes with the species information of the sequence in the $\mathrm{Nr}$ database (Ashburner et al. 2000). The intestinal microbial species of the black soldier fly larvae in different groups were counted in taxa of the kingdom, phylum, class, order, family, genus, and species. The gut larvae microbiota was abundant, reaching more than 2300 genera and 11,000 species. The number of microbial species on different taxa in different groups was relatively similar, but a significant difference was

Table 1 Assembled data assessment

\begin{tabular}{|c|c|c|c|c|c|c|}
\hline Sample & Total length (bp) & Contig number & Largest length (bp) & GC (\%) & N50 (bp) & Mapped (\%) \\
\hline$\overline{\mathrm{F} 1}$ & $713,374,913$ & 679,554 & 332,933 & 41.78 & 1115 & 97.07 \\
\hline F2 & $601,410,151$ & 594,324 & 264,723 & 41.58 & 1061 & 95.68 \\
\hline F3 & $621,800,601$ & 613,040 & 315,309 & 41.63 & 1065 & 95.84 \\
\hline $\mathrm{T} 1$ & $573,217,820$ & 519,415 & 184,590 & 41.85 & 1194 & 96.05 \\
\hline $\mathrm{T} 2$ & $716,521,041$ & 613,618 & 721,453 & 41.86 & 1290 & 97.17 \\
\hline T3 & $649,395,570$ & 557,814 & 186,681 & 42.01 & 1287 & 96.87 \\
\hline W1 & $604,749,023$ & 581,793 & 363,719 & 42.28 & 1072 & 95.93 \\
\hline W2 & $712,670,354$ & 668,870 & 399,518 & 42.11 & 1116 & 96.38 \\
\hline W3 & $680,722,887$ & 639,170 & 744,843 & 42.37 & 1108 & 96.35 \\
\hline
\end{tabular}

Sample is the sample number; total length is the sum of the base numbers of all contigs; contig numbers are the numbers of contigs after assembly; largest length is the number of bases in the longest contig; N50 are contigs that were sorted from long to short, and the cumulative length was counted. When a contig was added and the cumulative length was equal to half of the sum of the lengths of all contigs, the length of the contig was N50; Mapped is the alignment rate between sequencing reads and assembled contigs 
Table 2 Predicted genes overview

\begin{tabular}{llllll}
\hline Sample ID & Gene number & Total length (bp) & Average (bp) & Max length (bp) & Min length (bp) \\
\hline F1 & 550,158 & $152,574,384$ & 277 & 7536 & 102 \\
F2 & 464,889 & $125,423,319$ & 269 & 9972 & 102 \\
F3 & 485,384 & $132,226,104$ & 272 & 7536 & 102 \\
T1 & $117,609,837$ & 275 & 8286 & 102 \\
T2 & $152,545,194$ & 286 & 8175 & 102 \\
T3 & 531,807 & $132,783,018$ & 281 & 8904 & 102 \\
W1 & 472,169 & $164,800,824$ & 325 & 12,033 & 102 \\
W2 & 506,309 & $184,957,656$ & 319 & 10,866 & 102 \\
W3 & 579,598 & $195,543,927$ & 339 & 11,607 & 102 \\
Gene set & 576,423 & $608,038,053$ & 280 & 12,033 & 102 \\
\hline
\end{tabular}

Samples is the sample number; gene numbers is the number of predicted genes; total length is the base sum of predicted genes; average length is the average bases of predicted genes

observed in genus between groups $\mathrm{W}$ and $\mathrm{T}$. In general, the intestinal microbe species of group W were higher, but not in a significant level than those of groups $\mathrm{F}$ and $\mathrm{T}$ (Table 3).

\section{Analysis of high-abundant bacteria in the intestinal tract of black soldier fly larvae}

The resulting top 15 high abundance bacteria were selected for comparison. The results showed that the relatively high abundance of bacteria at the level of phylum and genus was highly comparable (Fig. 1). The highest relative abundance of bacteria belonged to Proteobacteria, Firmicutes, Bacteroidetes, and Actinobacteria phyla. The highest abundance of bacteria at the species level were Enterococcus, Acinetobacter, Providencia, Enterobacter, and Myroides.

\section{Similarity analysis of microbial and functional genes between groups}

The microbiota and functional gene composition of the samples were hierarchically clustered through $\mathrm{R}$ and the unweighted paired average method (UPGMA). Based on this, the similarity of species composition and functional gene composition of each sample was determined. The sample distance in the sample hierarchy clustering indicates the similarity of the species composition of the two samples. The results showed that samples in the same groups are closer and the branches are shorter; therefore, the microbial species structure and functional gene composition of the samples between the same treatment groups were comparable (Fig. 2a, c).

Principal component analysis (PCA) decomposes the differences of multiple sets of data on the twodimensional coordinate chart through processing complex data into two eigenvalues. Composition analysis of different samples (97\% similarity) reflects differences and distances between samples. The composition of the species in the two samples is similar, when they come closer on a PCA diagram. The results showed that the same sample in the same group was closer to each other, which demonstrated that the microbial composition and functional gene composition were comparable. The sum of the first dimension $(98.4 \%)$ and the second dimension (1.13\%) reached 99.53\%, which explains the difference between the different groups to a great extent (Fig. 2b, d).

\section{Analysis of differential microbes}

About 100 species were selected $(p<0.05)$ to draw differential heatmaps. According to the heatmap, there exists 35 high relative abundance species in group W (Faecalicatena contorta, Stenotrophomonas acidaminiphila, Sphingobacterium cellulitidis, Salana multivorans, Enterococcus sp. Gos25-1, Lachnospiraceae bacterium OF09-33XD, Pusillimonas caeni, Hungatella hathewayi, Sphingobacterium sp. 30C10-4-7, Stenotrophomonas sp. Leaf70, Pusillimonas sp. T7-7, uncultured Stenotrophomonas sp., Frischella perrara, Myroides sp. N17-2, Microbacterium sp. CH12i, Ochrobactrum sp. A44, Microbacterium ginsengiterrae, Kluyvera

Table 3 Microbial species statistics

\begin{tabular}{llllllll}
\hline Group & Kingdom & Phylum & Class & Order & Family & Genus & Species \\
\hline F & 6 & $115.33 \pm 1.15$ & $124.67 \pm 1.15$ & $269.67 \pm 1.53$ & $623.33 \pm 0.58$ & $2309.67 \pm 9.61 \mathrm{ab}$ & $11,510.33 \pm 25.50$ \\
$T$ & 6 & $115.33 \pm 1.53$ & $125.33 \pm 0.58$ & $269.33 \pm 2.08$ & $619 \pm 5.20$ & $2283 \pm 7.81 \mathrm{a}$ & $11,432.33 \pm 68.72$ \\
W & 6 & $111.67 \pm 1.15$ & $125.33 \pm 1.15$ & $269.67 \pm 0.58$ & $622 \pm 3.46$ & $2321.67 \pm 10.11 \mathrm{~b}$ & $11,880 \pm 17.09$ \\
\hline
\end{tabular}

Data are indicated ad means \pm standard error. Non-parametric ANOVA was used to analyze the data in the same column. Different letters in the same column indicate a significant difference $(p<0.05)$ between the groups 

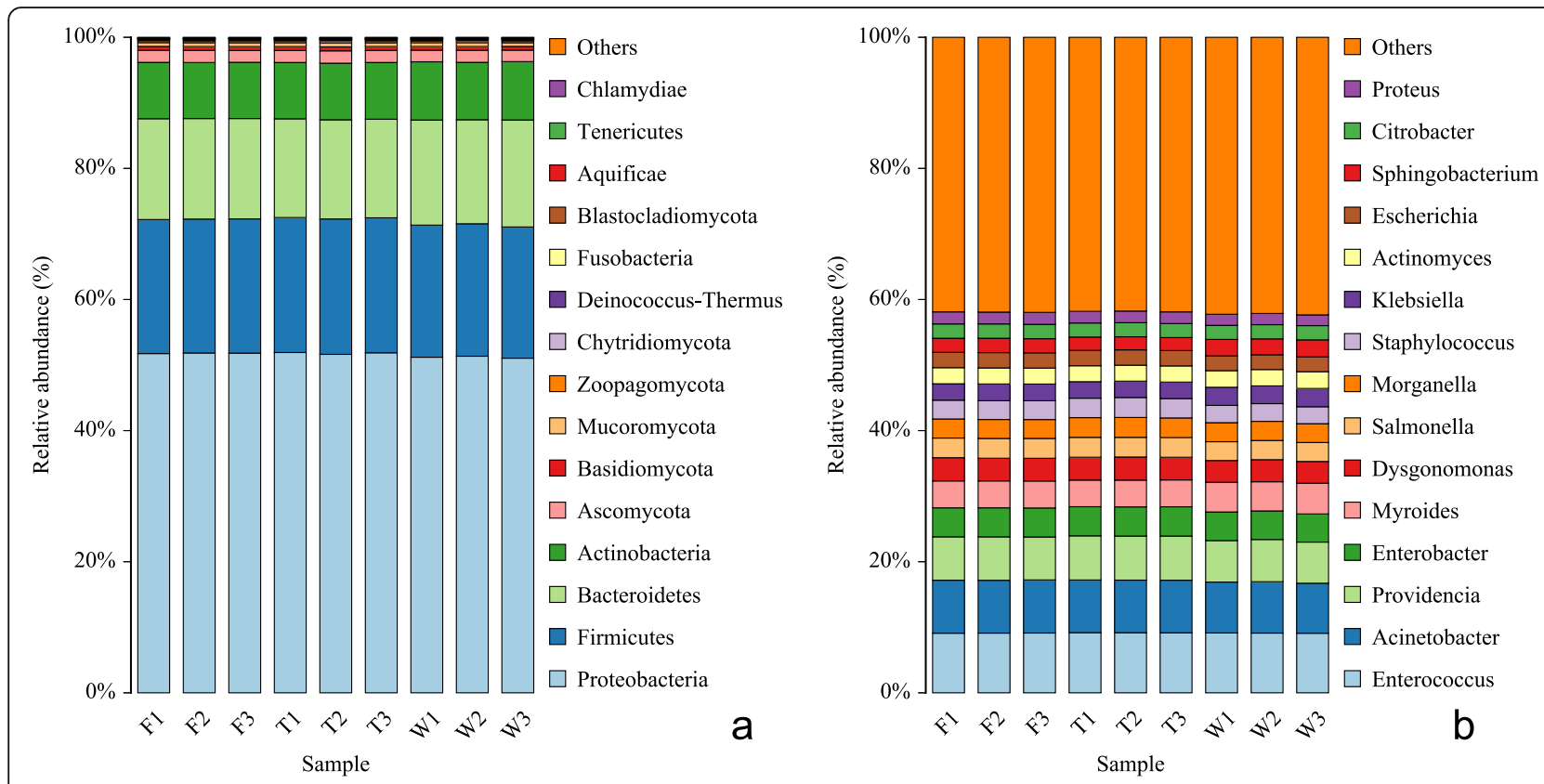

Fig. 1 Relative abundance microbiota analysis in the phylum and genus. a Relative abundance microbiota analysis in the phylum. b Relative abundance microbiota analysis in the genus. F1, F2, and F3 are replicates of group F; T1, T2, and T3 are replicates of group T; W1, W2, and W3 are replicates of group $\mathrm{W}$

georgiana, Sphingobacterium gobiense, Myroides odoratus, Klebsiella pneumoniae, Enterococcus pallens, Sphingobacterium lactis, Candidatus Schmidhempelia bombi, Aequorivita soesokkakensis, Vitellibacter aquimaris, Enterococcus sp. 9D6 DIV0238, Providencia stuartii, Miniimonas sp. PCH200, Gilliamella apicola, Orbus hercynius, Sphingobacterium mizutaii, Sphingobacterium sp. 1.A.4, Microbacterium profundi, Thermus filiformis), 34 in group $\mathrm{T}$ (Dysgonomonas capnocytophagoides, Allomyces macrogynus, Enterococcus sp. 9E7 DIV0242, Candidatus Erwinia dacicola, Enterococcus sp. 4G2 DIV0659, Propionibacteriaceae bacterium 16Sb5-5, Desulfovibrio sp. DS-1, Marinifilum breve, Tatumella sp. UCD-D suzukii, Bacillus velezensis, Staphylococcus gallinarum, Staphylococcus sciuri, Dysgonomonas sp. Marseille-P4361, Klebsiella aerogenes, Providencia rettgeri, Mycolicibacterium mucogenicum, Corynebacterium nuruki, Ruaniaceae bacterium KH17, Corynebacterium stationis, Enterococcus sp. 6C8 DIV0013, Enterococcus faecium, Providencia rustigianii, Wohlfahrtiimonas populi, Weissella jogaejeotgali, Bacillus amyloliquefaciens, Enterococcus casseliflavus, Bacteroides thetaiotaomicron CAG:40, Weissella thailandensis, Enterobacter hormaechei, Staphylococcus xylosus, Enterococcus saccharolyticus, Carnobacterium maltaromaticum, Spizellomyces punctatus), and 22 in group F (Citrobacter sp. MH181794, Schaalia canis, Metarhizium majus, Candida maltose, Nosocomiicoccus massiliensis, Lactobacillus sp. 54-5, Dysgonomonas gadei, Dorea longicatena, Prevotella sp. 10(H), Leminorella grimontii, Bacteroides caccae, Variovorax sp. EL159, Solibacillus isronensis, Proteus mirabilis, Actinomyces sp., Staphylococcus lentus, Rozella allomycis, Ignatzschineria sp. F8392, Leucobacter triazinivorans, Campylobacter concisus, Bacteroides sp. 11 14, Azospira oryzae). The relatively high abundance species of the different groups concentrate on different species without obvious overlap in the heatmap. Although the relatively high abundance species of the differential species in group $\mathrm{F}$ was the lowest, the relatively low abundance species were also less than the other two groups (Fig. 3).

\section{Analysis of differential functional gene}

The heatmap of different functional genes was obtained through a parametric test. The heatmap of the Kegg (Kanehisa et al. 2004) metabolic pathways in differential abundance gene shows that groups possess differences in polysaccharide biosynthesis and metabolism, translation, membrane transport, and energy metabolism. Group W results showed the highest relative abundance in four biological processes, group F maintained middlelevel abundance in those four-biological process, and group $\mathrm{T}$ showed low abundance in those four biological processes (Fig. 4a). The EggNOG (Powell et al. 2014) heatmap evidences the differences of the cytoskeleton, extracellular structures, inorganic ion transport and metabolism, nucleotide transport and metabolism, and coenzyme transport and metabolism in different groups. Group W showed a high abundance in extracellular 


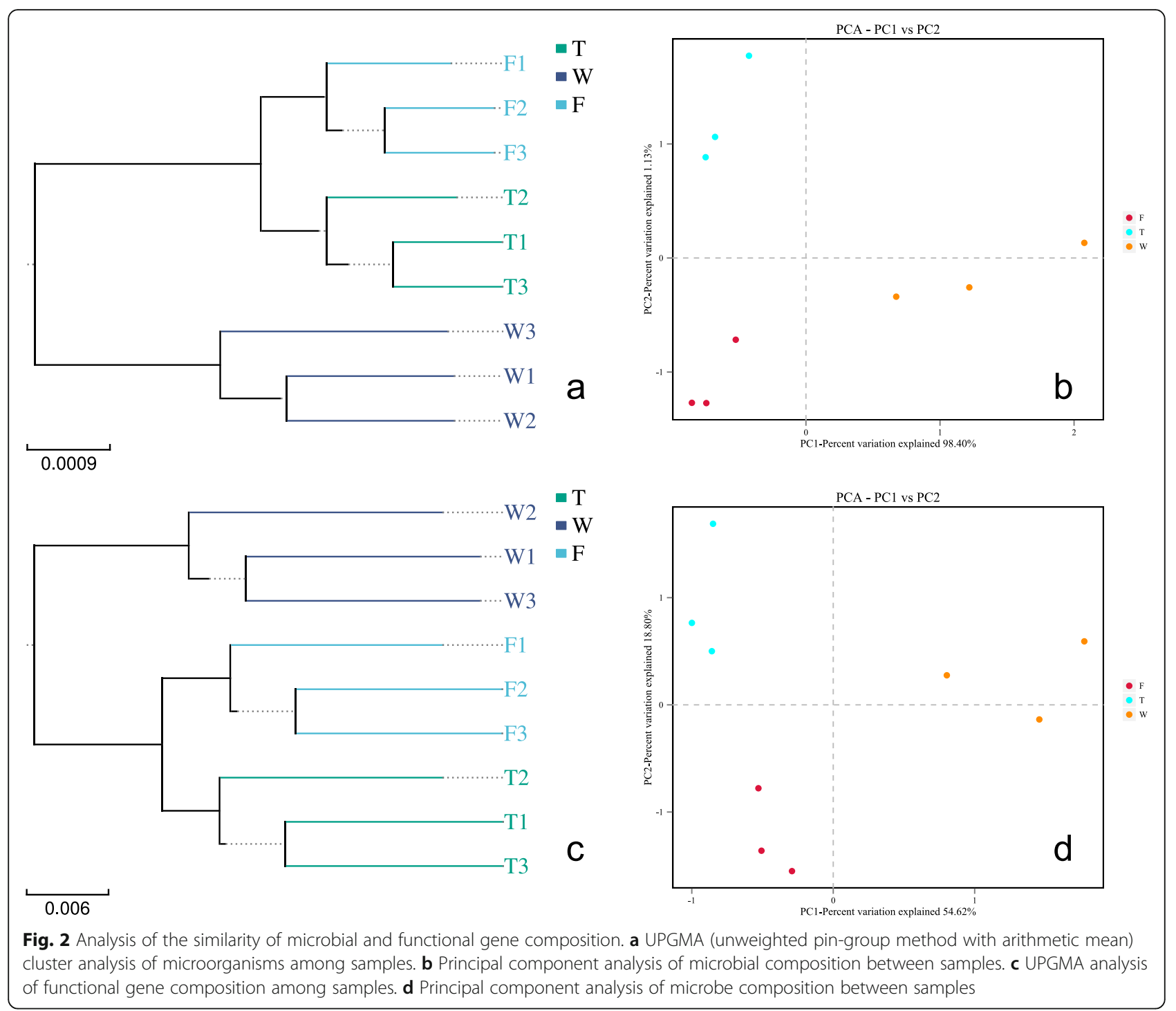

structure, inorganic ion transport and metabolism, nucleotide metabolism and transport, and coenzyme transport and metabolism, whereas the related functional genes of the cytoskeleton in group $\mathrm{T}$ showed relatively high abundance. The extracellular structures of group F were comparable to those of group W, whereas the other categories were less than those of group W (Fig. 4b).

\section{Discussion}

The N50 length of assembly sequence was about 1100 $\mathrm{bp}$, with an alignment rate exceeding $95 \%$, whereas the contig number of a single sample reached 600,000, which means that this data assembly quality meets the requirement of research and analysis. Gene number of a single sample up to 550,000 suggests that the sequencing depth is enough for subsequent microbial biological analysis. Moreover, the results of intestinal microbial statistics showed that there were over 11,000 intestinal bacteria in the black soldier fly larvae gut, indicating the presence of highly abundant microbes in the gut. It is relatively similar among different groups in each taxonomic order, indicating that different feeds had a limited influence on the overall microbial community. The top 15 high abundance microbes in the class and genus analysis results demonstrated that there was no apparent difference between the groups. The results indicate that feed played a slight role in the core gut microbiota of black soldier fly larvae, which was consistent with published research (Klammsteiner et al. 2020). The highest relative abundance bacteria belonged to the Proteobacteria, Firmicutes, Bacteroidetes, and Actinobacteria phyla. It showed some differences with Jeon et al.'s (2011) results, which included Bacteroidetes, Proteobacteria, Firmicutes, Fusobacteria, and Actinobacteria. Among those bacteria, Proteobacteria was observed as a potential microbial signature of disease, Firmicutes was 


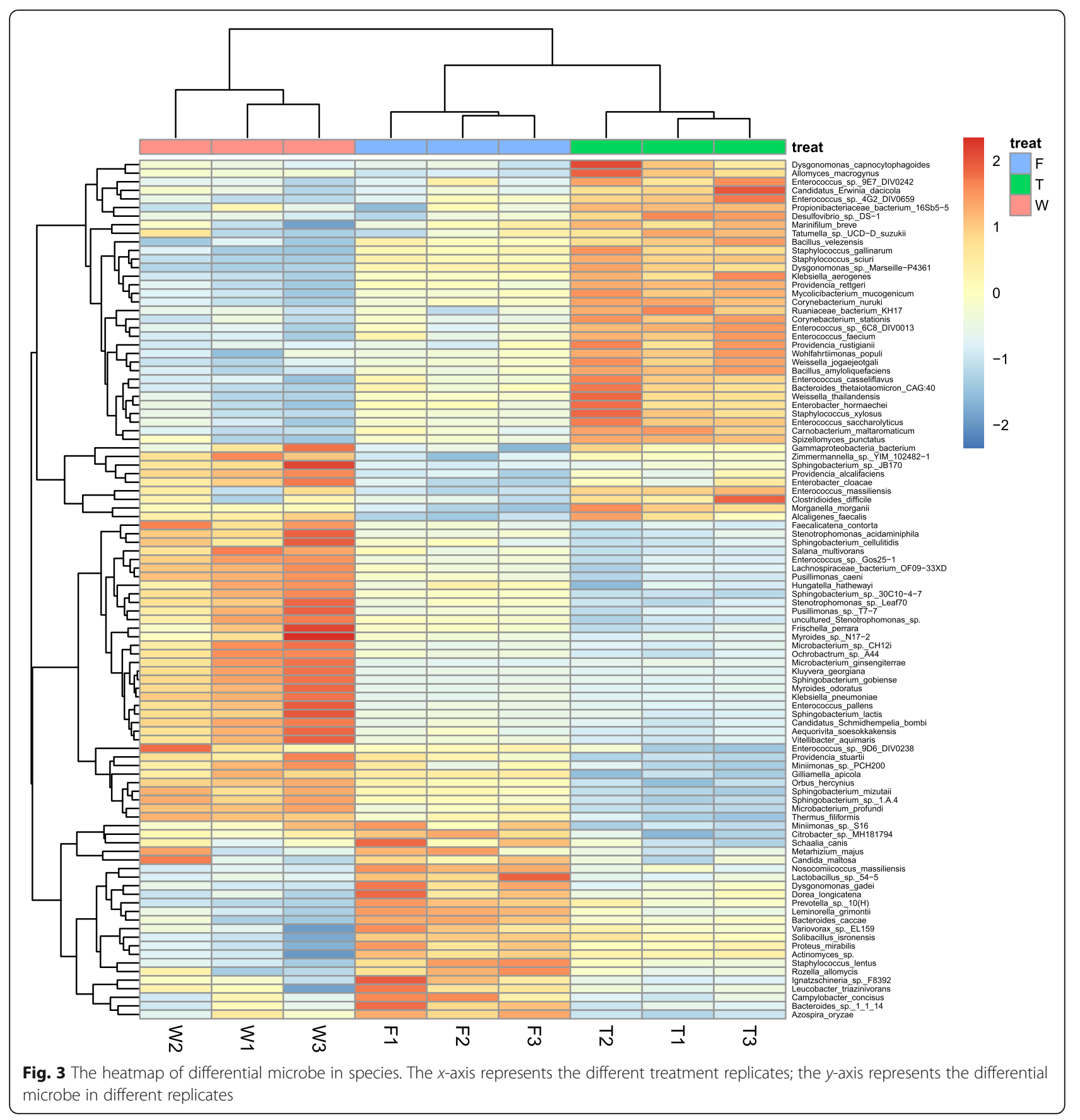

considered to play an important role in the digestion of animal manure, and Bacteroidetes were shown to degrade high-molecular weight organic matter (Zhan et al. 2020). Taken together, the larvae gut core microbiota facilitated degradation of animal manure and organic matter, which may provide microbial evidence for Kim et al.'s (2011) research. However, we should be more cautious to adopt black soldier larvae protein as edible for Proteobacteria in its gut (Wang and Shelomi 2017).
The intergroup similarity analysis of microbial species and functional genes demonstrates that both feeds and tetracycline had an impact on the intestinal microbial community structure of the black soldier fly larvae. The intestinal microbial community structure of larvae fed under a similar environment was comparable, which agrees with other studies (Jiang 2018; Tanaka et al. 2006; Liang et al. 2014; Bruno et al. 2019). Furthermore, our results agreed with (Engel et al. 2013) in that the diet 


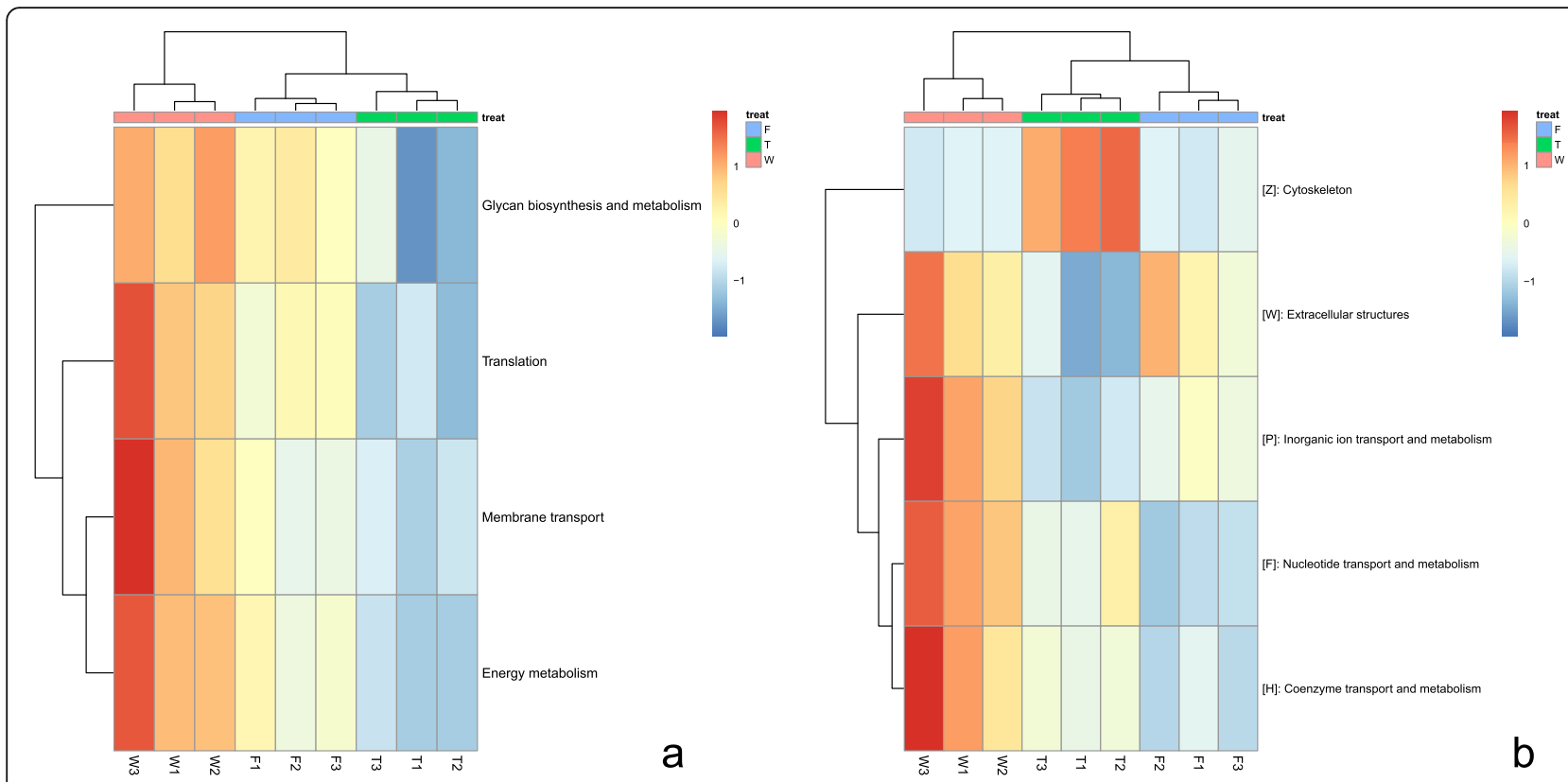

Fig. 4 Analysis of difference groups between functional genes. a Heatmap of differentially functional gene abundance in the Kegg metabolic pathway. $\mathbf{b}$ Heatmap of differentially functional genes in the eggNOG metabolic pathway. Genes shown have $p$ values $<0.05$, after metagenome sequencing analysis

shapes the composition and activity of the gut microbiota. Heatmap analysis results demonstrated that different treatments influence some microbes in the black soldier fly larvae. A similar trend shown in function genes indicates a relation between differential microbes and function genes. We can then infer that those microbes play a crucial role in black soldier fly larvae growth and development, which agrees with De Smet et al.'s (2018) conclusion. From these results, we may conclude the relatively high abundance microbiota in group W (35) and group F (22) play an important role in growth and development, whereas the relatively high abundance microbiota in group $\mathrm{T}$ (34) are relevant for black soldier fly larvae to survive in harsh conditions. However, further research is required to address such assumptions. Combining heatmap of Kegg and eggNOG analysis in differential function genes showed that relative function gene abundance of groups W, F, and T gradually reduces polysaccharide biosynthesis and metabolism, translation, and membrane transport and energy metabolism which associate with feeding. Hereby, we inferred that the 35 differential microbiota in group W were closely related to feeding. Furthermore, we believe that the differential microbiota in different groups play an important role in such biological process. Group $\mathrm{T}$ has a relatively high abundance of functional genes in the cytoskeleton indicating that the relatively high abundance of differential microbiota in group $\mathrm{T}$ was related to the cytoskeleton, which may be due to the inhibitory effect of tetracycline on some essential microbes involved in the development and feeding (Cai et al. 2018; Cifuentes et al. 2020), showing a great difference with mycotoxins and pesticides (Purschke et al. 2017). However, naval approaches are necessary to investigate this issue. The functional gene in group W displayed a high level in cell structure, inorganic ion transport and metabolism, nucleotide metabolic process, and coenzyme transport and metabolism, which indicate that the relatively high abundance of differential microbiota in group $\mathrm{W}$ is essential in these biological processes. Based on the aforementioned result, we conclude that the 35 differential microbes in group W were mainly associated with energy metabolism which influences growth a lot, whereas the 34 differential microbes in group $\mathrm{T}$ were related to survival in harsh environments. The present study provided a better understanding of the intestinal microbiota of black soldier fly larvae; however, it did not fully address the relation between microbes and function. We have research underway to elucidate such a relationship.

\section{Conclusion}

The present study demonstrated that gut bacteria in black soldier fly larvae were involved in polysaccharide biosynthesis and metabolism, translation, membrane transport, energy metabolism, cytoskeleton, extracellular structures, inorganic ion transport and metabolism, nucleotide metabolism, and coenzyme transport physiological processes. The 35 significant differential microbes in group W have a positive impact on energy metabolism and physiological 
process which could be exploited to improve transform efficiency. Subsequent studies will explore the function of specific differential high relative abundance microbes in group $\mathrm{W}$ and evaluate the possibility of improving farming efficiency with those microbiotas.

\section{Methods}

The research aims at exploring the relation between black soldier fly larvae and its intestinal microbiota. Fourth to 5th stage black soldier fly larvae fed with varied feeds were selected to study the intestinal microbiota. In order to avoid accidental outcomes, we set 3 repeats (10 individuals for 1 repeat) for every treatment. The metagenomic method which is a practical tool was utilized to achieve our goal.

\section{Insect sources and breeding}

Black soldier fly eggs and larvae were partly acquired from Guangzhou Anruijie Environmental Protection Technology Limited company, all of them were trapped in a field and reared in $25^{\circ} \mathrm{C}, 16 \mathrm{~h}$ photoperiod, $60-70 \%$ relative humidity feed for 30 generations.

After hatching from eggs, black soldier fly larvae were incubating with $75 \%$ wheat bran, $25 \%$ soybean powder (group W), food waste (group F), or $75 \%$ wheat bran and $25 \%$ soybean powder supplemented with $1 \%$ tetracycline (group T) for 10 days. Then, 10 4-5th stage larvae were selected in each group for intestinal anatomy, performing triplicate determinations.

\section{Intestinal dissection of black soldier fly larvae}

Black soldier fly larvae were starved for $24 \mathrm{~h}$, after which they were washed with sterile water and inactivated for $10 \mathrm{~min}$ at $-20^{\circ} \mathrm{C}$. Next, they were surface sterilized with $75 \%$ alcohol and washed with sterile water. Larva intestines were dissected out with sterile scissors and tweezers on a sterile operating table, eliminating intestinal adhesions. The obtained larvae intestines were preserved at $-80^{\circ} \mathrm{C}$ for subsequent sequencing at Beijing Biomarker Technologies.

\section{Metagenomic sequencing and bioinformatics analysis}

DNA isolation, sequencing, and bioinformatics analysis were performed by Beijing Biomarker Technologies, acquiring $10 \mathrm{G}$ of sequencing data for every replicate (three replicate determinations were achieved). Filtering and quality controlling were processed to get original clean reads for subsequent analysis. The Trimmomatic software was used for original splicing sequences (raw tags), whereas Bowtie2 was used to sequence alignment with the host genome to remove host contamination. MEGAHIT (Gurevich et al. 2013) was utilized for metagenome assembly, and contig sequences shorter than $300 \mathrm{bp}$ were not considered. QUAST (Zhu et al. 2010) was used to evaluate assembly results, whereas MetaGeneMark (Fu et al. 2012) was used to predict the encoding genes and perform functional annotation on the encoding genes in general database and special database. Those bioinformatics analysis tools were kept in default parameter while analysis performing. Taxonomic analysis was performed based on clean reads data. Furthermore, species composition, abundance information, and function genes of the samples were statistically analyzed.

\section{Statistical analyses}

The statistics in this research were analyzed in Prism GraphPad 8; the figures were drawn through R.

\section{Acknowledgements \\ We thank Beijing Biomarker Technologies for providing the data analysis services. The authors would like to express their gratitude to EditSprings (https://www.editsprings.com/) for the expert linguistic services provided.}

\section{Authors' contributions}

All authors contributed to the study conception and design. The first draft of the manuscript was written by Yuan Zhineng, and all authors commented on previous versions of the manuscript. All authors read and approved the final manuscript.

\section{Funding}

The National Key R\&D Program of China (grant number 2017YFF0210204) supported this research but was not involve in the design of the study; collection, analysis, and interpretation of the data; and writing of the manuscript.

\section{Declarations}

Ethics approval and consent to participate

No approval of research ethics committees was required to accomplish the goals of this study because experimental work was conducted with an unregulated invertebrate species. The larvae were utilized to experiment without abuse or maltreatment. All authors understand that my participation is voluntary and agree to participate in the above research.

Consent for publication

Written informed consent for publication was obtained from all participants.

\section{Competing interests}

The authors declare that they have no competing interests.

\section{Author details}

${ }^{1}$ The School of Life Sciences, Sun Yat-sen University, Guangzhou 510275, China. ${ }^{2}$ Guangdong Agribusiness Tropical Agriculture Institute, Guangzhou 510000, China. ${ }^{3}$ The School of Agriculture, Sun Yat-sen University, Guangzhou 510275, China.

Received: 27 November 2020 Accepted: 22 February 2021 Published online: 12 March 2021

\section{References}

Ashburner M, Ball CA, Blake JA et al (2000) Gene ontology: tool for the unification of biology. Nat Genet 25(1):25-29

Basset A, Khush RS, Braun A et al (2000) The phytopathogenic bacteria Erwinia carotovora infects Drosophila and activates an immune response. PNAS 97(7): 3376-3381

Beskin KV, Holcomb CD, Cammack JA et al (2018) Larval digestion of different manure types by the black soldier fly (Diptera: Stratiomyidae) impacts associated volatile emissions. Waste Manage 74:213-220

Bruno D, Bonelli M, Filippi FD et al (2019) The intestinal microbiota of Hermetia illucens larvae is affected by diet and shows a diverse composition in the different midgut regions. Appl Environ Microbiol 85(2):e01864-e01818 
Cai M, Ma S, Hu R et al (2018) Systematic characterization and proposed pathway of tetracycline degradation in solid waste treatment by Hermetia illucens with intestinal microbiota. Environ Pollut 242:634-642

Ceja-Navarro JA, Karaoz U, Bill M et al (2019) Gut anatomical properties and microbial functional assembly promote lignocellulose deconstruction and colony subsistence of a wood-feeding beetle. Nat Microbiol 4(5):864-875.

Cifuentes Y, Glaeser SP, Mvie J et al (2020) The gut and feed residue microbiota changing during the rearing of Hermetia illucens larvae. Antonie Van Leeuwenhoek 113(9):1323-1344

De Smet J, Wynants E, Cos P et al (2018) Microbial community dynamics during rearing of black soldier fly larvae (Hermetia illucens) and impact on exploitation potential. Appl Environ Microbiol 84(9):e02722-e02717

Douglas AE (1998) Nutritional interactions in insect-microbial symbioses: aphids and their symbiotic bacteria Buchnera. Annu Rev Entomol 43:17-37

Engel P, Moran NA (2013) The gut microbiota of insects - diversity in structure and function. FEMS Microbiol Rev 37:699-735.

Fu LM, Niu BF, Zhu ZW et al (2012) CD-HIT: accelerated for clustering the nextgeneration sequencing data. Bioinformatics 28(23):3150-3152

Furrie $E$ (2006) A molecular revolution in the study of intestinal microflora. Gut 55(2):141-143

Gavriel S, Jurkevitch E, Gazit Y et al (2011) Bacterially enriched diet improves sexual performance of sterile male Mediterranean fruit flies. J Appl Entomol 135(7):564-573

Gurevich A, Saveliev V, Vyahhi N et al (2013) QUAST: quality assessment tool for genome assemblies. Bioinformatics 29(8):1072-1075

Hammer TJ, Bowers MD (2015) Gut microbes may facilitate insect herbivory of chemically defended plants. Oecologia 179(1):1-14

Handelsman J (2004) Metagenomics: application of genomics to uncultured microorganisms. Microbiol Mol Biol Rev 68(4):669-685

Hehemann JH, Correc G, Barbeyron T et al (2010) Transfer of carbohydrate-active enzymes from marine bacteria to Japanese gut microbiota. Nature 464(7290): 908-912

Hoyt CP, Osborne GO, Mulcock AP (1971) Production of an insect sex attractant by symbiotic bacteria. Nature 230(5294):472-473

Jeon H, Park S, Choi J et al (2011) The intestinal bacterial community in the food waste-reducing larvae of Hermetia illucens. Curr Microbiol 62(5):1390-1399

Jiang CL (2018) Effect of gut microbiome of black soldier fly larvae (Hermetia illuences) on the biodegradation of food waste. Dissertation, Zhejiang University, Zhejiang

Kanehisa M, Goto S, Kawashima S et al (2004) The kegg resource for deciphering the genome. Nucleic Acids Res 32(suppl 1):D277-D280

Kim W, Bae S, Park K et al (2011) Biochemical characterization of digestive enzymes in the black soldier fly, Hermetia illucens (Diptera: Stratiomyidae). J Asia-Pac Entomol 14(1):11-14

Klammsteiner T, Walter A, Bogataj T et al (2020) The core gut microbiome of black soldier fly (Hermetia illucens) larvae raised on low-bioburden diets. Front Microbiol 11:993

Lee C-M, Lee Y-S, Seo S-H (2014) Screening and characterization of a novel cellulase gene from the gut microflora of Hermetia illucens using metagenomic library. J Microbiol Biotechnol 24:1196-1206

Lee Y-S, Seo S-H, Yoon S-H (2016) Identification of a novel alkaline amylopullulanase from a gut metagenome of Hermetia illucens. Int J Biol Macromol 82:514-521

Li GN, Xia XJ, Tang WC et al (2016) Intestinal microecology associated with fluoride resistance capability of the silkworm (Bombyx mori L.). Appl Microbiol Biotechnol 100(15):6715-6724

Li Q, Zheng LY, Cai H et al (2011) From organic waste to biodiesel: black soldier fly, Hermetia illucens, makes it feasible. Fuel 90(4):1545-1548

Liang X, Fu Y, Tong L et al (2014) Microbial shifts of the silkworm larval gut in response to lettuce leaf feeding. App Microbiol Biotechnol 98(8):3769-3776

Nguyen TT, Tomberlin JK, Vanlaerhoven S (2015) Ability of black soldier fly (Diptera: Stratiomyidae) larvae to recycle food waste. Environ Entomol 44(2): 406-410

Park SI, Kim JW, Yoe SM (2015) Purification and characterization of a novel antibacterial peptide from black soldier fly (Hermetia illucens) larvae. Dev Comp Immunol 52(1):98-106

Powell S, Forslund K, Szklarczyk D et al (2014) eggNOG v4.0: nested orthology inference across 3686 organisms. Nucleic Acids Res 42(Database issue):231239

Prado S, Almeida R (2009) Role of symbiotic gut bacteria in the development of Acrosternum hilare and Murgantia histrionica. Entomol Exp Appl 132(1):21-29
Purschke B, Scheibelberger R, Axmann S (2017) Impact of substrate contamination with mycotoxins, heavy metals and pesticides on growth performance and composition of black soldier fly larvae (Hermetia illucens) for use in the feed and food value chain. Food Addit Contam Part A Chem Anal Control Expo Risk Assess 34:1410-1420

Rehman KU, Rehman A, Cai MM et al (2017) Conversion of mixtures of dairy manure and soybean curd residue by black soldier fly larvae (Hermetia illucens L.). J Cleaner Prod 154:366-373

Shelomi M, Wu MK, Chen SM, Burke CG et al (2020) Microbes associated with black soldier fly (Diptera: Stratiomiidae) degradation of food waste. Environ Entomol 49(2):405-411

Tanaka H, Aoyagi H, Shiina S et al (2006) Influence of the diet components on the symbiotic microorganism's community in hindgut of Coptotermes formosanus Shiraki. Appl Microbiol Biotechnol 71(6):907-917

van Huis A (2013) Potential of insects as food and feed in assuring food security. Annu Rev Entomol 58:563-583

Wang YS, Shelomi M (2017) Review of black soldier fly (Hermetia illucens) as animal feed and human food. Foods 6:91

Warnecke F, Luginbuhl P, Ivanova N et al (2007) Metagenomic and functional analysis of hindgut microbiota of a wood-feeding higher termite. Nature 450(7169):560-565

Xie JF (2010) The screening and Identification of cellulase bacterial strain from intestines of Hermetia illueens L. Maggots. Dissertation, Huazhong Agricultural University, Wuhan

Zhan S, Fang G, Cai M et al (2020) Genomic landscape and genetic manipulation of the black soldier fly Hermetia illucens, a natural waste recycler. Cell Res 30 : $50-60$

Zhu W, Lomsadze A, Borodovsky M (2010) Ab initio gene identification in metagenomic sequences. Nucleic Acids Res 38(12):132-132

\section{Publisher's Note}

Springer Nature remains neutral with regard to jurisdictional claims in published maps and institutional affiliations.
Ready to submit your research? Choose BMC and benefit from:

- fast, convenient online submission

- thorough peer review by experienced researchers in your field

- rapid publication on acceptance

- support for research data, including large and complex data types

- gold Open Access which fosters wider collaboration and increased citations

- maximum visibility for your research: over $100 \mathrm{M}$ website views per year

At $\mathrm{BMC}$, research is always in progress.

Learn more biomedcentral.com/submissions 